\title{
Regenerative Medicine Using Platelet Rich Plasma and Stem Cells in Atrophic Acne Scars: A Case Report
}

Pradeep V Mahajan, Juzer Abbasi ${ }^{\star}$, Swetha Subramanian, Siddhesh C Parab and Amit Danke

Stem Rx Bioscience Solutions Private Limited, Mumbai, India

*Corresponding author: Juzer Abbasi, Stem Rx Bioscience Solutions Pvt. Ltd., Level 3, Block-13, Andheri East Mumbai, India, Tel: +919821299952; E-mail: jzrabbasi@yahoo.co.in

Received date: April 20, 2017; Accepted date: May 29, 2017; Published date: June 05, 2017

Copyright: ( 2017 Mahajan PV. This is an open-access article distributed under the terms of the Creative Commons Attribution License, which permits unrestricted use, distribution, and reproduction in any medium, provided the original author and source are credited.

Keywords: Sebum production; Hormonal dysfunction; Bacterial activity; Inflammation; Granulation

\section{Introduction}

Acne is a distressing condition for physicians and patients alike. Over $90 \%$ adolescents acquire acne with $12-14 \%$ cases persisting even in adulthood. The condition and its' sequelae may cause long term psychological disturbance to the patient as well as compromise in physical appearance $[1,2]$.

Etiologic factors such as increased sebum production, hormonal dysfunction, bacterial activity within the follicle (commonly Propionibacterium acnes) etc. are responsible for occurrence of acne [3]. Acne healing process progresses through stages of follicular inflammation, granulation tissue formation and finally matrix remodelling $[4,5]$. Scarring occurs due to skin damage during healing of active acne. Depending on the loss or gain of collagen, acne scars may be broadly classified as atrophic and hypertrophic, with atrophic scars being more common.

Conventional line of treatment of acne and scars involve measures to reduce duration and intensity of inflammation. Anti-scar medications may aid in prevention of hypertrophic scars and keloids but do little in treating atrophic scars. Such modalities, though effective to certain extent, have long downtime and may be associated with side effects in darker skin types [6].

Maintenance of dermal homeostasis and regeneration is a function of stem cells of the epidermis and hair follicles. These stem cells are also known to participate in wound healing [7]. Research on cell based therapy is now showing promise in treatment of various dermatological conditions. Stem cells have high proliferative potential and are capable of self-renewal. Anti-fibrotic, anti-inflammatory, antiapoptotic as well as angiogenic properties of mesenchymal stem cells [8] may be beneficial in treatment of atrophic acne scars [9]. Mesenchymal stem cells derived from adipose tissue was shown to enhance the effects of fractional carbon dioxide resurfacing for skin rejuvenation as well as for the treatment of atrophic acne scars especially in lighter skin types [10].

Additionally, Platelet-rich plasma (PRP) which is an autologous preparation of platelets in concentrated plasma may benefit scar healing by promoting collagen deposition by providing sustained release of growth factors [11]. Multipotency of mesenchymal stem cells is thus an attractive option for treatment of patients with acne scars. In the current scenario, many aestheticians and aesthetic surgeons prefer the use of platelet rich plasma alone or incombination with other modalities of treatment to improve atrophic acne scars of the face irrespective of skin type [12].
In the present report, a combination technique using mesenchymal stem cells and platelet rich plasma for the treatment of atrophic acne scars in a female patient with a darker skin type has been described.

\section{Case Report}

A 25 year old female Indian patient came to our clinic with chief complaints of:

Scars on face for more than 1 year,

Hyper pigmented marks on face,

History of moderate acne since 4 years,

Occasional pustules with pus discharge,

Acne flare-up during menstrual cycle and after consumption of oily foods.

Significant family history was observed wherein the patient's father and older brother suffered from similar lesions of acne. Medical history revealed that the patient was treated in the past with diuretics for renal calculi. However, she had never undergone dermatological treatment for acne or scars.

\section{Local examination revealed}

Type 5 (darker skin type): Fitzpatrick Skin type.

Type 2 (moderate) photoaging: Glogau photo aging classification.

Grade 3 (moderate acne scarring): Global Acne Scarring Classification.

Acne lesions in the form of papules and few pustules were seen. Ice pick, Boxcar and Rolling type of atrophic acne scars were seen bilaterally (Figure 1).
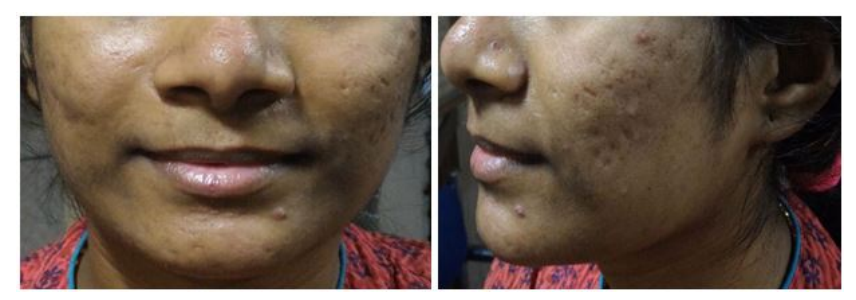

Figure 1: Anterior view and lateral view before treatment. 
After thorough clinical and hematological investigations, the patient was advised three sessions of autologous stem cell therapy with platelet rich plasma. The treatment protocol for each session was as follows:

Priming of bone marrow was done by administering granulocyte colony stimulating factor at a dose of $5 \mu \mathrm{g} / \mathrm{kg} /$ day for 2 consecutive days before treatment, $80 \mathrm{cc}$ of bone marrow and $60 \mathrm{cc}$ of adipose tissue were aspirated from iliac crest and gluteal region respectively under aseptic precautions during each treatment session. $50 \mathrm{cc}$ of peripheral blood was aspirated from cubital vein during each session of treatment, the collected samples were taken to a Regenerative Medicine Laboratory where isolation and culture of cells was done, peripheral blood was centrifuged and supernatant platelet rich plasma was separated.

Therapeutically effective volume of bone marrow and adipose derived mesenchymal stem cells and platelet rich plasma was transplanted intradermally covering all lesions on the face. An average of 500-5000 $\times 10^{6}$ bone marrow cells, $1600-4000 \times 10^{6}$ adipose cells, and $1.5 \times 10^{6}$ platelet rich plasma was transplanted.

Injection Glutathione $(600 \mathrm{mg})$ and Vitamin C (1 g) was administered intravenously once a week. Topical application of aloevera extract containing copper peptide $\{2 \mathrm{ml}$ ( $0.1 \%$ concentration) of solution in $120 \mathrm{ml}$ of cream $\}$ was advised daily along with mild massaging of treated area.

\section{Results}

After one session of therapy, fading of hyperpigmentation marks was noted and the patient's face looked more supple (Figure 2). Healing of acne pustules and papules was also noted. Reduction in prominence of boxcar and rolling acne scars was observed following second session of therapy. A transition to Grade 1 (mild acne scars) was noticed after the third session of therapy the results of which have been maintained till date (Figure 3).
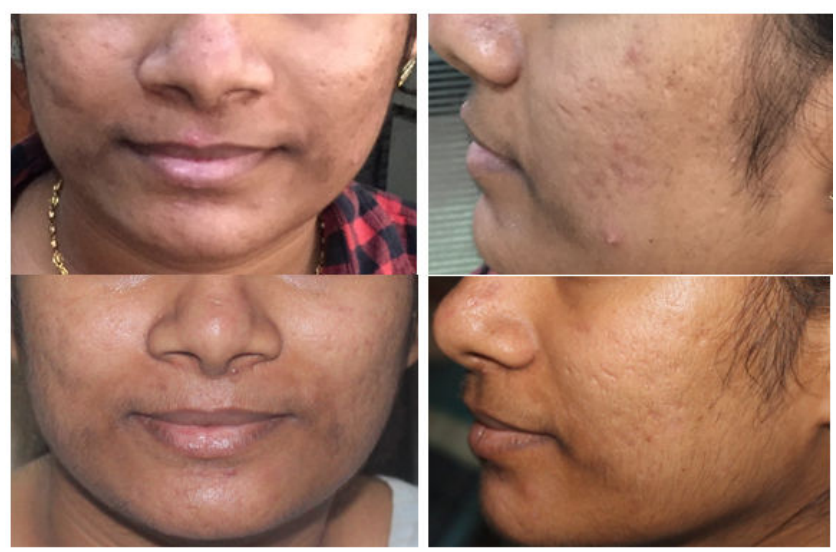

Figure 2: Top left and right denote results after $1^{\text {st }}$ session of therapy; Bottom left and right denote changes after $2^{\text {nd }}$ session.

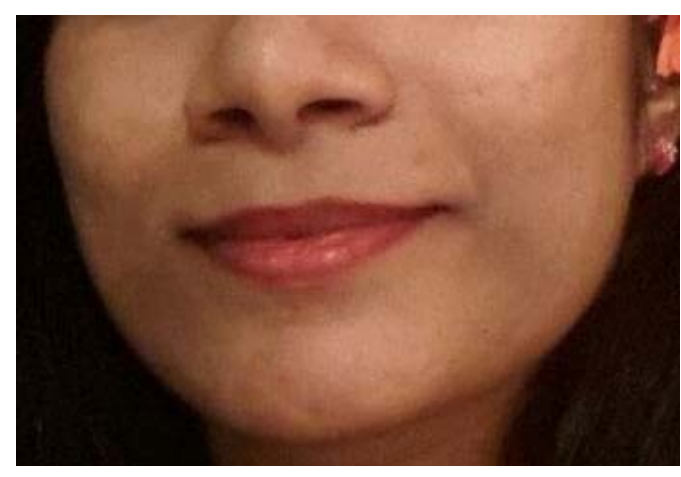

Figure 3: Notice the improvement in scars after $3^{\text {rd }}$ (final) session of treatment.

\section{Discussion}

Multiple treatment options (medical and surgical) are available for treatment of acne scars, however, no general guidelines exist. Most conventional treatments are associated with long healing time and limited efficacy outcomes. This case report documents a case of moderate acne scars that has been treated effectively using mesenchymal stem cells and PRP therapy.

Mesenchymal stem cells are capable of self-renewal as well as differentiation into various tissues, including epidermis and other skin appendages. The various properties of these cells are therefore promising in treatment of various conditions affecting the human body.

The result of treatment of in this study was in consensus with those from other studies done on atrophic acne scars. [9] in a pilot study on 14 patients treated with intradermal bone marrow MSCs, found significant improvement in the qualitative and quantitative grading as well as Cardiff Acne Disability Index (CADI) scores of acne scars with no adverse effects.

MSCs derived from adipose tissue have been shown to support formation of new vascular networks through self assembly of transplanted cells and endothelial progenitor cells. Restoration of tissue vascularisation offers high potential for skin regeneration and maintenance of skin homeostasis. [9] in 2016 treated 13 patients with atrophic acne scars using mesenchymal stem cells derived from adipose tissue. The study reported increased subject satisfaction, skin hydration as well as skin elasticity. Histology study showed increased dermal collagen and elastin density.

Platelet rich plasma contains growth factors such as, PDGF, VEGF, TGF-beta, EGF, FGF and IGF-1. PRP secrete $70 \%$ of the stored growth factors within 10 minutes and close to $100 \%$ within the first hour. Thereafter additional amounts of growth factors are synthesized up to 8 days [13]. This sustained release of growth factors supplements the activity of mesenchymal stem cells by enhancing collagen deposition thereby resulting in improved treatment outcomes.

Asif, et al. in 2016 enrolled fifty atrophic acne scar patients aged 17 to 32 years [1]. Intradermal injections along with topical application of platelet rich plasma was given to all patients on right half of the face, while their left half of the face was treated with intradermal administration of distilled water only. The patients underwent three 
consecutive treatment sessions at one month intervals. Goodman's Qualitative scale was used for assessment of treatment outcomes which showed better outcomes on the right side $(40 \%)$ as compared to left (16\%). The authors concluded that platelet rich plasma has excellent efficacy in management of atrophic acne scars and should be combined with other therapies in order to achieve better treatment outcomes.

Similar positive treatment outcome was reported by [11] where statistically significant improvement in the degree of atrophic acne scars was observed after intradermal injection of PRP in 15 patients.

As previously described, inflammation, subsequently resulting in oxidative stress has been implicated as an etiologic factor in acne [14]. Antioxidants such as glutathione and vitamin $\mathrm{C}$ were administered to the patient in this study in order to diminish the burden of oxidative stress and enhance the effects of cell based therapy. Similarly, copper peptide used in this study is a naturally occurring copper complex of amino acids which has anti-inflammatory and epidermal stem cell enhancing properties. Therefore, the role of nutraceuticals is to help the stem cells achieve a favorable environment to show everlasting regenerating effects [15]. The result of combination therapy of mesenchymal stem cells and platelet rich plasma along with nutraceuticals used in this study are being maintained remarkably and has enhanced not only the physical appearance but also emotional wellbeing of the patient.

\section{Conclusion}

Accelerated wound healing, skin regeneration and other properties of mesenchymal stem cells play an important role in treatment of atrophic acne scars. Autologous cell based therapy being minimally invasive is an effective and safe therapeutic modality for atrophic acne scars. Regenerative medicine approach with the aid of nutraceuticals may be an attractive treatment option for patients who do not wish to undergo or are not candidates for conventional therapy. Further studies done on larger population groups may define precisely the role played by various cells in dermatological conditions, thereby enabling formulation of newer cell based therapy protocols.

\section{Competing Interests}

The authors declare that there is no conflict of interests regarding the publication of this paper.

\section{References}

1. Asif M, Kanodia S, Singh K (2016) Combined autologous platelet-rich plasma with microneedling verses microneedling with distilled water in the treatment of atrophic acne scars: a concurrent split-face study. J Cosmet Dermatol 15: 434-443.

2. Fabbrocini G, Annunziata MC, D'arco V, De Vita V, Lodi G, et al. (2010) Acne scars: pathogenesis, classification and treatment. Dermatol Res Pract 2010: 893080.

3. Kurokawa I, Danby FW, Ju Q, Wang X, Xiang, LF, et al. (2009) New developments in our understanding of acne pathogenesis and treatment. Exp Dermatol 18: 821-832.

4. Wolfram D, Tzankov A, Pulzl P, Piza-Katzer H (2009) Hypertrophic scars and keloids-a review of their pathophysiology, risk factors, and therapeutic management. Dermatol Surg 35: 171-181.

5. Cowin AJ, Brosnan MP, Holmes TM, Ferguson MW (1998) Endogenous inflammatory response to dermal wound healing in the fetal and adult mouse. Dev Dyn 212: 385-393.

6. Goodman GJ, Baron JA (2006) Postacne scarring: a qualitative global scarring grading system. Dermatol Surg 32: 1458-1466.

7. Blanpain C, Fuchs E (2006) Epidermal stem cells of the skin. Annu Rev Cell Dev Biol 22: 339-373.

8. Lee DE, Ayoub N, Agrawal DK (2016) Mesenchymal stem cells and cutaneous wound healing: novel methods to increase cell delivery and therapeutic efficacy. Stem Cell Research \& Therapy 7: 37.

9. Ibrahim ZA, Eltatawy RA, Ghaly NR, Abd El-Naby NM, Abou El Fetouh HM, et al. (2015) Autologus bone marrow stem cells in atrophic acne scars: A pilot study. J Dermatolog Treat 26: 260-265.

10. Zhou BR, Zhang T, Bin Jameel AA, Xu Y, Guo SL, et al. (2016) The efficacy of conditioned media of adipose-derived stem cells combined with ablative carbon dioxide fractional resurfacing for atrophic acne scars and skin rejuvenation. J Cosmet Laser Ther 18:138-148.

11. Nofal E, Helmy A, Nofal A, Alakad R, Nasr M (2014) Platelet-rich plasma versus CROSS technique with $100 \%$ trichloroacetic acid versus combined skin needling and platelet rich plasma in the treatment of atrophic acne scars: a comparative study. Dermatol Surg 40: 864-873.

12. Tenna S, Cogliandro A, Barone M, Panasiti V, Tirindelli M, et al. (2017) Comparative Study Using Autologous Fat Grafts Plus Platelet-Rich Plasma With or Without Fractional $\mathrm{CO}_{2}$ Laser Resurfacing in Treatment of Acne Scars: Analysis of Outcomes and Satisfaction With FACE-Q. Aesthetic Plast Surg 41: 661-666.

13. Lubkowska A, Dolegowska B, Banfi G (2012) Growth factor content in PRP and their applicability in medicine. J Biol Regul Homeost Agents 26: $3 S-22 S$.

14. Lopez-Estebaranz JL, Herranz-Pinto P, Dreno B (2017) Consensus-Based Acne Classification System and Treatment Algorithm for Spain. Actas Dermosifiliogr 108: 120-131.

15. Marx RE (2001) Platelet-rich plasma (PRP): what is PRP and what is not PRP? Implant Dent 10: 225-228. 